\title{
Mathematics in Paradise
}

\author{
by Lizhen Ji \\ Department of Mathematics, University of Michigan
}

\section{The idea of master lectures on mathematics}

What is Paradise? Where is Paradise? What do people do in Paradise?

One answer is to listen to master lectures on mathematics in Sanya! From January 4 to January 8, 2013, inside a beach front hotel in the Sanya bay, hundreds of people from all over the world were actively participating in four

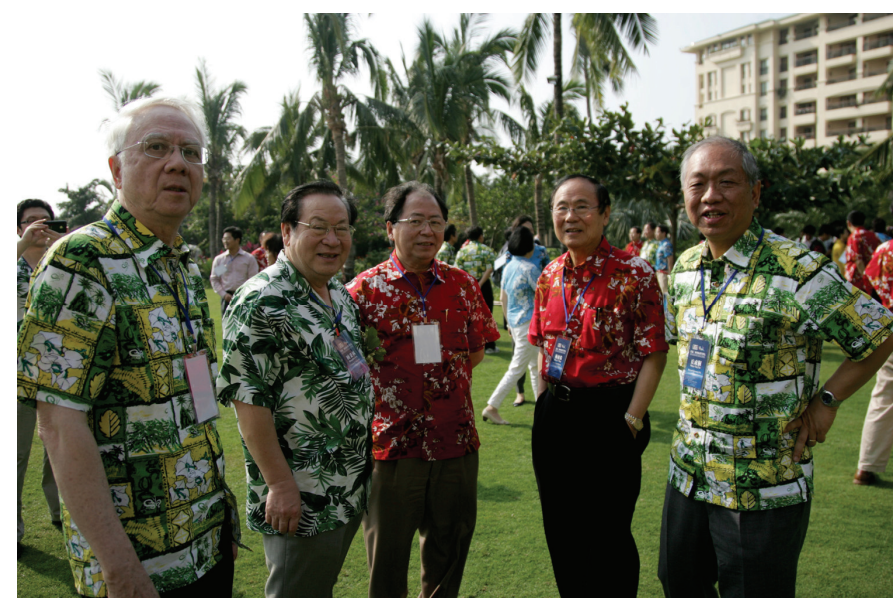

Left to right: C. L. Liu (the former President of National Tsing Hua University), Binglin Gu (the former President of Tsinghua University), Lih-Jun Chen (the president of National Tsing Hua University), Henry T Yang (Chancellor of the University of California, Santa Barbara), Shing-Tung Yau (Harvard University). Photo taken in 2010, Sanya.

workshops on contemporary mathematics.

These workshops were proceeded by five plenary talks by five masters in mathematics: David Mumford (Brown University, Fields Medalist), Richard Schoen (Stanford University), Wilfried Schmid (Harvard University), Stanislav Smirnov (University of Genava, Fields Medalist), and Shing-Tung Yau (Harvard, Fields Medalist).

Why do we start these master lectures? What do we want to achieve?

Mathematics has a long history. It is exciting and beautiful, but is also sometimes complicated and myste- rious for beginners. To keep the river of mathematics forever flowing, we need the new blood and the new generation. Yes, the future belongs to them.

In order to encourage and educate the future mathematicians, we started this conference titled "Master Lectures on Mathematics." Its purpose is to ask masters in mathematics to show the young what great mathematics is, how it is discovered, what the excitement and pain in the creative process are, what are waiting to be discovered (or the future directions of mathematics). Of course, this will also add a humanistic dimension to our endeavors. Mathematics is created by mathematicians, and mathematicians are people too. The interaction of the human beings forms the civilization.

The conference series "Master Lectures on Mathematics" will be held once a year during the winter break in

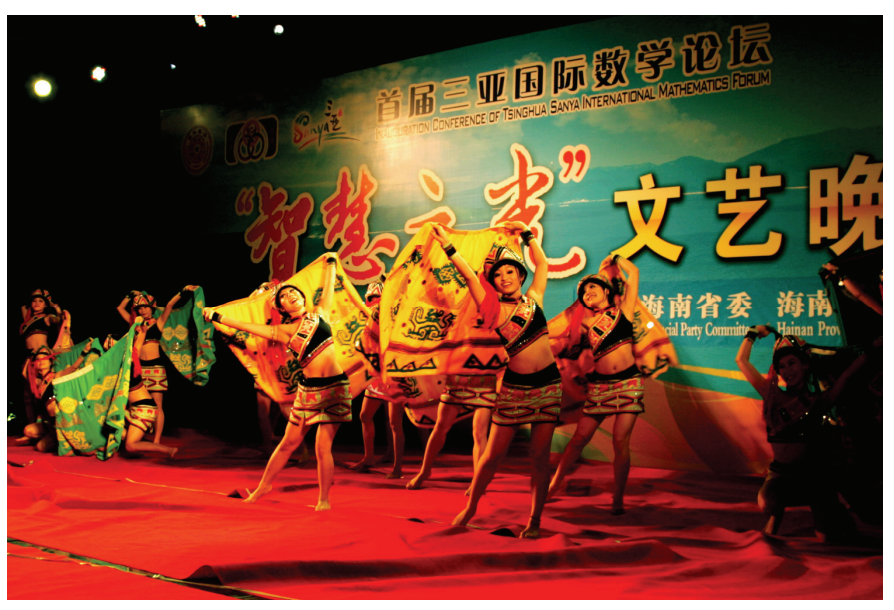

The First Tsinghua-Sanya International Mathematics Forum, 2010

the tropical Sanya city.

We believe that such a conference series will convey the idea that mathematics is as exciting as ever, more useful than ever before, and will continue to play a pivotal role in the further development of science. We hope and also strongly believe that it will have a big impact on the mathematics community by providing a platform for the young people to interact with the masters in mathematics. 
Tsinghua Sanya International Mathematics Forum is a new conference center of Tsinghua University, one of the leading universities in China. Tsinghua University has a long tradition in mathematics and has educated generations of Chinese mathematicians including S. S. Chern and L. K. Hua. Several distinguished mathematicians from the West such as Wiener and Hadamard visited and taught at Tsinghua University. The interaction between the West and China has had a huge impact on the development of mathematics in China. Tsinghua Sanya International Mathematics Forum is a special conference center devoted to mathematics, theoretical physics and related fields and will play a pivotal role in the future of mathematics.

\section{Wandering on the Sanya beach}

What did people talk about at this conference?

Yau gave a talk titled "Geometry: from Riemann to Einstein and onto String Theory" which explores the his-

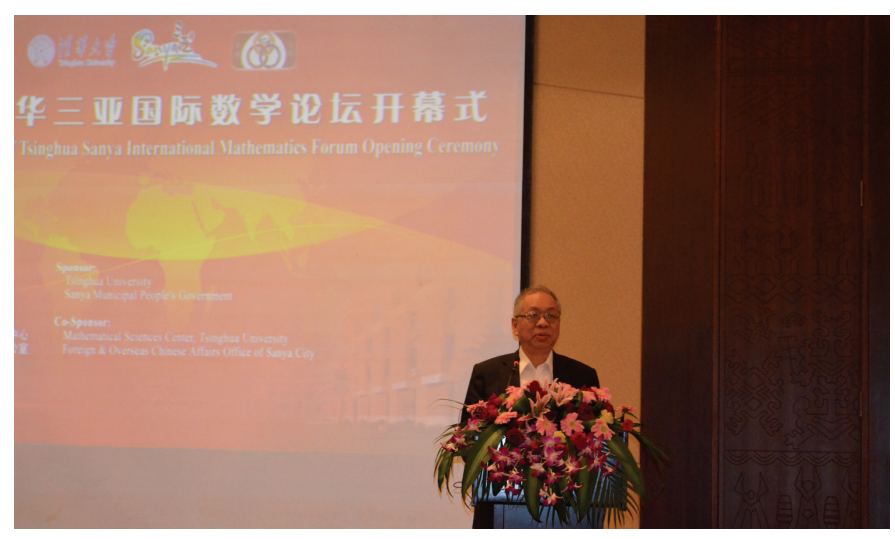

Shing-Tung Yau, 2013 at Sanya

tory of geometry and its inseparable interaction with the never-ending search for unifying theories of the universe. Sanya is probably the most suitable place to give and listen to such a talk, since it is so close to the nature and one can see and feel the motion and attraction of the universe. Early in the morning, one can walk on the beach and swim in the ocean, and one can feel the gentle touch of the waves and watch the steady rise of the Sun. Questions and answers flow in and out of one's mind. The wind may cause the waves, but what is responsible for the tides? It is the gravity (or the pull) from the moon. How to unify the waves with the gravity? This is one of the topics of Yau's lecture, a theory which unifies the quantum theory and the gravity through the vibration of strings. Where do the strings dance? In the space-time compactified by Calabi-Yau manifolds.

The gentle waves make the morning beach even more quiet, and the night tides compactify the sand. Does it resemble the Calabi-Yau compactification? Maybe yes, maybe no. Maybe it is a good place and a time to think the meaning of life, the love of life, or even better the question of what mathematics is.

What is pure mathematics? And what is applied mathematics? The public lecture by Mumford dealt with "The Partnership of Pure and Applied Mathematics." Is it not clear that the pure mathematics and applied mathematics are different? But is there really a clear dividing line between them? Though all waves look similar and one follows another, each wave is different. Many times one thinks that one can keep the pants dry, but a sudden high wave breaks the expectation. Yes, every mathematician thinks differently too about what is pure, what is non-pure, and what is good and important mathematics. Many things have changed over time, but waves have been hitting the beach in the same way forever. Mumford said that the idea of modern computing was used by the ancient Chinese thousands of years ago!

There are fine and rough sands. How many grains of sand are there on the Sanya beach? Maybe no one has ever asked this question, maybe no one has ever cared about it. Yes, probably no one should care about each grain of sand. Each grain of sand moves randomly and its fate cannot be predicted. It is the statistic behavior of grains of sand which counts. No matter how fine the sand is, the beach surface is not smooth. It is packed with small balls. How does one do analysis on the beach, or on a discrete surface? Smirnov's lecture was titled "Discrete complex analysis and probability." It discusses the interplay between the discrete and the continuous. How does the wet line on the beach form and change? Maybe the percolation theory in his talk can help.

In the evening, the Sanya beach is still lively with motor rides and karaoke singers. But the beauty of the nature can not be hidden by the dark sky. Under the beach umbrella and on the beach chair, one can lie down and listen to the harmonious sound of the ocean waves. Maybe it is the time to also think about mathematics discussed during the day time. What is harmony in nature? What is the harmony in music? What is the harmonic analysis in mathematics? Schmid gave a talk on "Harmonic analysis on Lie groups." Beauty depends on symmetry, and the mathematics of symmetry is the group theory. What is the connection between the beauty of the Sanya coastline and the group theory? Yes, the group of self-similarity explains the mystery of fractals, which explain for the formation of coastlines.

Walking barefoot on the beach is fun and enjoyable, especially when the sand is smooth. The sand is smooth when each grain is round. Why are grains of sand round? It is the effect of time, the persistent washing or kissing of the ocean waves. Perhaps it is the mean curvature flow which is more responsible to make the sand round and smooth. The mean curvature does not only appear in the sand but also in soap-bubbles (maybe also in the wave fronts?). Yes, it is needed in the minimal surfaces. Schoen gave a talk on "Minimal submanifolds in differential geometry." The classical subject is full of new life and sur- 
prises, and the nature always seeks the most stable, minimal energy state.

Building on the plenary talks of these masters, the four workshops in Sanya covered the most recent developments in Vision Science, Geometric Analysis, Representation Theory, and Planar Statistical Models.

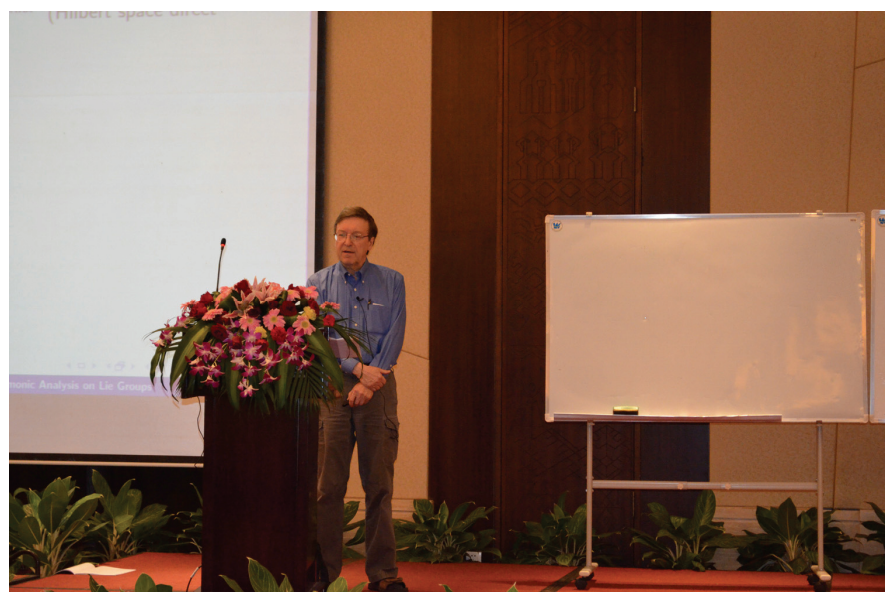

Wilfried Schmid

Rich mathematical diets are intertwined with exotic tropical fruits during the breaks, and the conference was enjoyable and a success.

\section{Encounter between masters and students}

From whom should one learn? Abel said, "Learn from Masters!"

Besides researchers from around the around, there

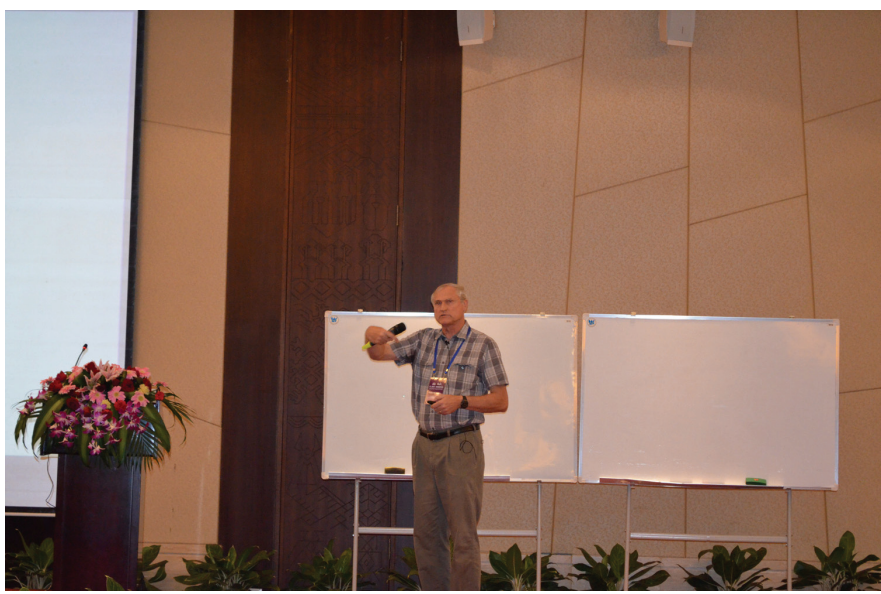

Richard Schoen

were also selected undergraduate students and graduate students from Tsinghua University and various universities in China. They dream of the future and own the future.

Some mathematics talks might over their head, but the experience of listening to masters will stay in their memory forever.
It is even better to ask the masters how they became masters. In the evening of January 7, the top students from the top universities engaged the top mathematicians from the West in a lively panel discussion, or a dialogue

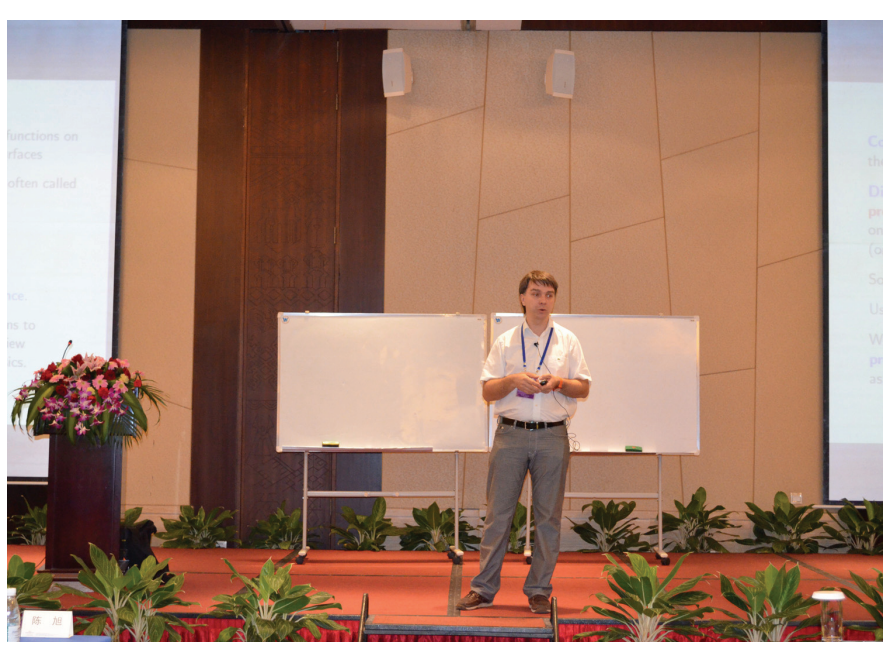

Stanislav Smirnov

titled "Encounter with the masters."

Is there a single event in their life which changed their mathematical life?

It depends. It could be the resolution of a simple singularity, or the beauty and simplicity of the plane geometry, or meeting the right mathematics tutor at a young age.

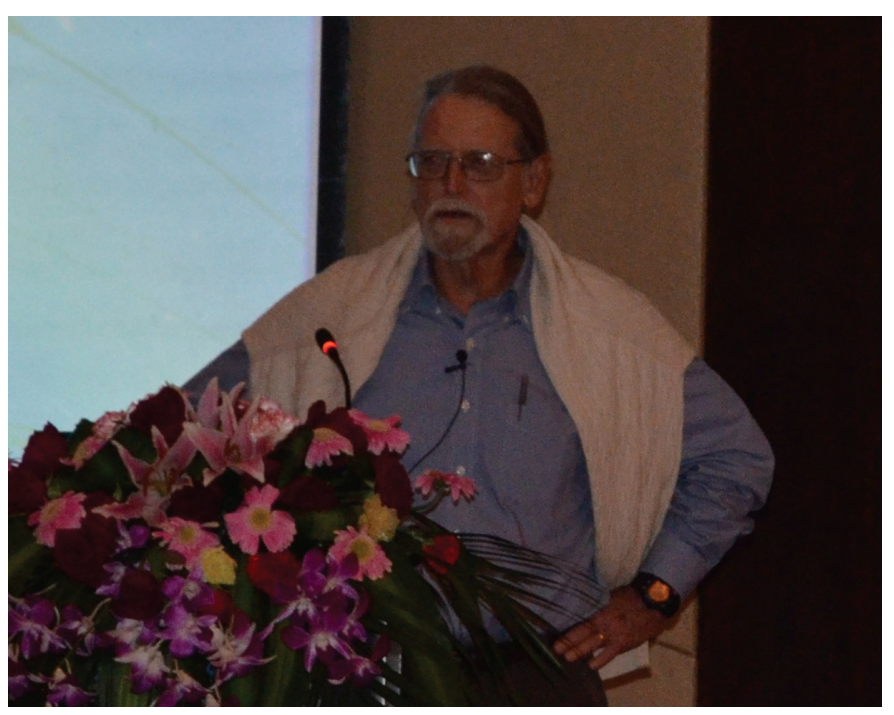

David Mumford

How to read books, listen to talks, talk to people, choose a right subject and a right problem? Should it be a hot topic? Should it be a basic one?

There are no easy answers.

Is it always joy in studying mathematics? How to overcome pain of no progress, no understanding, or failure? 
No, life is not easy, which makes it more interesting. There is no much geometry on a single straight line. Twisting and unexpected turns produce a rich subject.

The interaction between the West and the East has been going on for a long time and will continue. But each encounter is unique.

What is the difference between the education and study in the West and the East? What is the advice for the Chinese students going to the West?

The West is not homogeneous, and it depends where you go. Maybe the West will come to the East in the future.

The final advice is that to choose and study something you like (i.e., pursue your love) and be persistent, and you will have a rewarding life!

\section{Third Conference of Tsinghua Sanya International Mathematics Forum}

The Third Conference of Tsinghua Sanya International Mathematics Forum was organized by Shing-Tung Yau (chair), Shiu Yuen Cheng, Yat Sun Poon, Jie Xiao and Lizhen Ji.

It was held at the Howard Johnson Resort in Sanya Bay from January 5 to January 8, 2013 and consisted of the following four workshops together with five plenary talks mentioned above:

1. Vision, organized by David Mumford, David Xianfeng $\mathrm{Gu}$, Song-Sun Lin, and Song-Chun Zhu.

2. Geometric Analysis, organized by Richard Schoen, Yng-Ing Lee, and Mu-Tao Wang.

3. Representation Theory, organized by Wilfried Schmid, Shouwu Zhang, Dragan Milicic, Stephen D. Miller, Ye Tian, and Xinyi Yuan.

4. Planar Statistical Models, organized by Stanislav Smirnov, Hugo Duminil, Clément Hongler, Ka-Sing Lau, Vladas Sidoravicius, and Yuefei Wang.

More detailed description of the workshops such as titles and abstracts is available at the website: http://msc.tsinghua.edu.cn/forum2013/Default.aspx

Besides many speakers and participants from around the world, over thirty undergraduate and graduate students from Tsinghua University and over twenty students from other universities were selected and came to the conference, and they interested with the masters and other speakers. It was a rewarding experience for both the students and the masters. 